\title{
Gregorio Marañón en el Instituto de Medicina Legal, Toxicología y Psiquiatría Central de España
}

\author{
Gregorio Marañón in the Legal Medicine Institute, \\ Center of Toxicology and Psychiatry of Spain \\ J. D. Villalaín Blanco \\ Catedrático de Medicina Legal \\ M. T. Ramos Almazán \\ Profesor Titular de Medicina Legal
}

\begin{abstract}
Resumen
Prácticamente, todos los biógrafos de Gregorio Marañón ignoran los trece años (1912-1925) en que trabajó en el Instituto de Medicina Legal, Toxicología y Psiquiatría Central de España. Una experiencia previa que tuvo que repercutir necesariamente en la metodología y el buen hacer de nuestro polígrafo.
\end{abstract}

Los autores a partir de las más diversas fuentes tratan de analizar esa fase de la biografía de Marañón, actualmente olvidada en general y especialmente por la Medicina Legal.

\section{Palabras claves}

Gregorio Marañón. Instituto de Medicina Legal, Toxicología y Psiquiatría.

\section{Summary}

Virtually all biographers of Gregorio Marañón ignore the thirteen years in which he worked in the Legal Medicine Institute, Center of Toxicology and Psychiatry of Spain. A previous experience (1912-1925) which must have had repercussions in our writers' methodology and work. Authors using the most diverse sources try to analyze that part of Marañón's biography, currently forgotten and especially by Legal Medicine.

\section{Key words}

Marañón, Legal Medicine Institute, Toxicology and Psychiatry. 


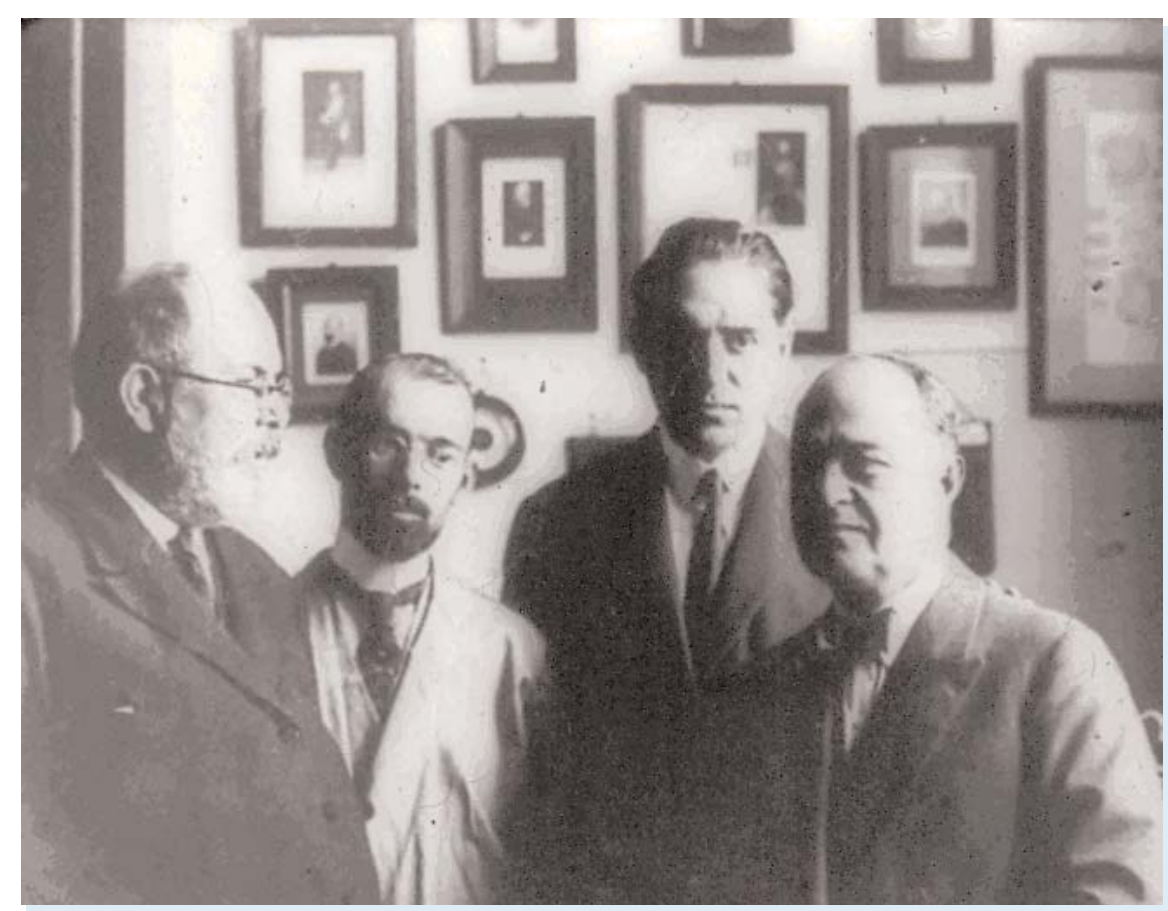

Fotografía 1 Gregorio Marañón, junto a un profesor Sudamericano, al Prof. Maestre y al Prof. Ara en los locales del antiguo Instituto de Medicina Legal Toxicología y Psiquiatría, en la Facultad de Medicina de San Carlos

Tenemos que reconocer que la figura de Marañón, uno de los grandes intelectuales del siglo pasado, ha ejercido siempre una evidente fascinación, especialmente en el ambiente universitario general y en el medico-sanitario en particular. Durante nuestros estudios de Medicina, siendo alumno interno en el Hospital Provincial de Madrid, aún tuvimos ocasión de asistir a alguna de las últimas de sus famosas sesiones clínicas, que nos dejaron un recuerdo imborrable. Su fama, ese mínimo contacto y la lectura de sus obras acentuó nuestro interés por su figura y magisterio.

En el último año de la licenciatura, siendo alumno interno de la Cátedra de Medicina Legal de la Universidad Complutense, nos llamó la atención que, tanto en la Cátedra como en la Escuela de Medicina Legal, donde se integraba, existían referencias sobre la asistencia de Marañón al Instituto de Medicina Legal Toxicología y Psiquiatría, organismo antecedente de la Escuela de Medicina Legal, que había fundado uno de los grandes Maestros de la Medicina Legal Española: el Prof. Maestre. Posteriormente, siendo profesor encargado de Curso, y profesor Ayudante, utilizando el poco material existente procedente de la antigua sede de la Escuela de Medicina Legal en la Facultad de Medicina de San Carlos de la calle de Atocha, realizamos un pequeño Museo, en los largos pasillos del tercer piso que se le habían asignado a la Escuela de Medicina Legal en el pabellón IV, dentro de la Facultad de Medicina, en la Ciudad Universitaria. Al recoger el posible material para esta exposición, y entre la colección de fotografías que procedía de la exposición con que se inauguró la Escuela de Medicina Legal por Alfonso XIII, nos llamó la atención una fotografía en la que aparece Gregorio Marañón, junto a un profesor Sudamericano, al Prof. Maestre y al Prof. Ara en los locales del antiguo Instituto de Medicina Legal Toxicología y Psiquiatría, en la Facultad de Medicina de San Carlos (Fotografía 1).

Posteriormente, con motivo del nuevo traslado de la Cátedra y Escuela de Medicina Legal, en la misma Facultad de Medicina, desde un pabellón a otro, nos encontramos otro documento, igualmente revelador, que se había utilizado por el reverso: papel impreso con el membrete del Instituto, con la leyenda: 
«Instituto de Medicina Legal. Prof. Maestre, Sección de Medicina Experimental, Director: Profesor G. Marañón, Prof. G. Carrasco Cadenas y Prof. A. Soler». (Fotografía 2). Este documento se complementó con otra cuartilla también reaprovechada en que bajo el epígrafe general anterior, se podía leer: Sección de Biología: Gregorio Marañón. Quiere esto decir, que por un período, todavía a concretar, D. Gregorio Marañón mantuvo una intensa actividad científica y organizadora medicolegal en que dirigió una Sección de Medicina Experimental y se encargo de desarrollar las funciones propias de la Sección de Biología del Instituto de Medicina Legal, Toxicología y Psiquiatría Central de España.

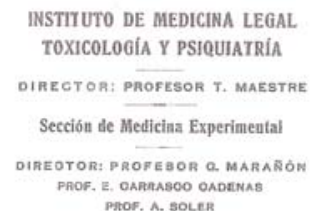

Sr. D.

Fotografía 2 Papel impreso con el membrete del Instituto, con la leyenda: «Instituto de Medicina Legal. Prof. Maestre, Sección de Medicina Experimental, Director: Profesor G. Marañón, Prof. G. Carrasco Cadenas y Prof. A. Soler».

Tratamos de complementar estos pocos datos, a través de la consulta de las biografías al uso, sin que en ninguna se cite detalladamente esta época y actividad; sin embargo, la participación de Gregorio Marañón en trabajos medicolegales ha quedado plasmada en las declaraciones realizadas por otros autores, especialmente por Teófilo Hernando, a quien también tuvimos la suerte de conocer en su fructífera longevidad, y las de Pérez de Petinto.

Teófilo Hernando fue uno de los grandes maestros de la Medicina, que tuvo una permanente amistad y colaboración con Marañón. Hernando, obtuvo en 1906 su quinta oposición: de auxiliar de terapéutica y medicina legal. Desde este puesto conoció a Marañón, que así comenzó siendo alumno suyo para, con el devenir del tiempo convertirse en amigo inseparable. La penúltima oposición de Hernando fue la de médico forense, que consiguió en 1909; dos años después marchó a Estrasburgo con una beca de la Junta para la Ampliación de Estudios, para mejorar su formación farmacológica, para, en 1912, obtener la Cátedra de Terapéutica.

La amistad entre Hernando y Marañón se inicia, por lo tanto, a través de la relación profesor-alumno en Medicina Legal; Marañón asistió a sus clases cuando, siendo auxiliar Hernando impartía las prácticas de Medicina Legal; después explicándole algunas clases teóricas y examinándole finalmente de la asignatura en tribunal compartido con Tomás Maestre (mereció Marañón la calificación de sobresaliente y sabemos que mereció una entusiastita felicitación de D. Tomás). También se sabe que Marañón acompañaba muchas veces a Hernando cuando éste practicaba autopsias en su calidad de médico forense. En una de estas ocasiones es cuando D. Teófilo y Marañón ven por primera vez unas glándulas suprarrenales. A partir de ahí progresó el mutuo conocimiento que cristalizó en una sólida amistad que se confirmó con la edición del célebre Manual de Medicina Interna. Este libro se publicó cuando Hernando tenía 35 años y Marañón 27. La guerra acentuó esta unión en el exilio de París, donde coincidieron, 
El propio Hernando recuerda estos principios en una entrevista que le hizo hace años Fernández-Braso con motivo de su 91 cumpleaños: «Conocí a Marañón cuando siendo auxiliar de Medicina Legal asistió a un cierto número de mis lecciones y a las autopsias que yo practicaba, Nuestra amistad no fue demasiado cultivada hasta que un día, en el otoño del 14, al salir de San Carlos, me acompañó en dirección a mi casa»... «Aunque he tenido amistad con tantos y tan diversos grandes hombres, la que me unió a Gregorio Marañón fue entrañable, y no ya mantenida, sino acrecentada a lo largo de los años, del que presumo haber sido hasta confidente».

El profesor Rafael Calvo expresa agudamente esta amistad: «Estas dos vidas, verdaderamente paralelas, nada ni nadie separó nunca hasta la muerte de Don Gregorio, que tan profundamente conmovió a Hernando. Si unidos y compenetrados estuvieron en días dichosos de los triunfos y de las conquistas para la ciencia, lo estuvieron también en las horas tristes de la adversidad».

En el ámbito medico-forense he podido leer un párrafo revelador que apareció en el Boletín de la Asociación Nacional de Médicos Forenses que dirigía Pérez de Petinto: «El Prof. D. Teófilo Hernando Ortega, como Presidente Nacional del Homenaje a Gregorio Marañón, solicita suscripción entre los compañeros para allegar fondos precisos, que permitan la erección del monumento a Marañón: encargado al escultor Pablo Serrano (realizado por Ochoa). En su día será instalado en la plaza central de la Ciudad Universitaria, frente a la Facultad de Medicina.

La gloriosa memoria de Marañón quien, en su inicial carrera, estuvo vinculado al Primitivo Instituto de Medicina Legal, Psiquiatría y Toxicología que fundó en 1914 el Dr. D. Tomás Maestre. Y la solicitud, por quien es maestro también de todos, por D. Teófilo: antiguo y magnífico Médico Forense de Madrid (hasta la conquista de su cátedra de Terapéutica, tan acertadamente continuada por B. Lorenzo Velázquez)...». Los médicos forenses de España aportaron 5.000 pesetas al monumento.

Esta participación de Marañón en la Medicina Legal, ha dejado muy pocos rastros en su copiosa producción literaria. Sin embargo, en su catálogo de publicaciones existen cuatro representativas de esta tendencia., independientemente de otras que tienen evidentemente implicaciones medicolegales. Las dos primeras correspondientes a este primer período en que Marañón aporta su concepto y vivencia de lo que es la Medicina Legal y las otras dos, mucho más tarde, demostrativas de su permanente contacto continuado con este área. La primera, que apareció en la revista Justicia, del año 1911, se titulaba "La Medicina Legal y las Nuevas Orientaciones de la Ciencia»; la segunda, que firmó el año 1912 con Claudio Aznar, que era profesor del Instituto de Medicina Legal, y se titula «La Acción Protectora de Diferentes Extractos de Órganos Contra la Estricnina», trabajo que implica su necesaria presencia en el laboratorio de Toxicología del Instituto de Medicina Legal.

Las otras dos publicaciones a que nos referíamos son del año 1936 y corresponden a la serie de sus prólogos. Uno que inicia el libro del conocido profesor de Medicina Legal brasileño Leonidio Ribeiro «Homosexualismo y Endocrinología», y que Marañón denomina una clasificación de los homosexuales desde el punto de vista medicolegal. Y otro de introducción a un librito de Piga Pascual titulado «Terapéutica de los Gaseados de Guerra», prólogo que agradece tanto Piga, que dedica el libro al propio Marañón.

Resulta también muy reveladora su relación con su condiscípulo Salvador Pascual, que, no lo olvidemos, cuando se constituyó la actual Escuela de Medicina Legal en el año 29, era Jefe de la Sección de Toxicología y poco después Subdirector de dicha Escuela. Marino Gómez-Santos recoge estas rela- 
ciones y refiere cómo el Rey utilizaba al Dr. Pascual, médico del Príncipe de Asturias, para obtener información de Marañón en los sucesos del año 31.

Una referencia directa a ese periodo la encontramos en el prólogo al libro de Salvador Pascual «Supuraciones renales», con el título «Una generación y un hombre». En ese trabajo glosa la amistad que le unía con el autor del modo siguiente: «Hice con él todos los estudios académicos y ni un solo instante nos hemos separado después. Su orientación difería de la mía. Pero, aparte de la amistad, nos reunieron, durante largos años, los trabajos en común en el laboratorio de Medicina Legal de Madrid, bajo la blanda dirección de don Tomás Maestre; la colaboración en aquella inolvidable Revista Clínica de Madrid, en que pusimos tantos entusiasmos y donde ambos nos entrenamos para la literatura científica; y varias ocasiones más en que coincidió nuestro esfuerzo...».

Las buenas relaciones que Marañón mantuvo con el sector medicolegal se aprecian en el apoyo positivo que le dieron Maestre, como Director de la Escuela de Medicina Legal, y Barahona, como Catedrático de Medicina Legal, a la propuesta de la Cátedra de Endocrinología de doctorado, que le concedieron en 1931 tras haber fundado el Instituto de Patología y construido el pabellón que lo albergaba en el Hospital Provincial.

Tuvimos ocasión de conocer, por nuestro trabajo como alumno interno de la Cátedra, en la citada Escuela de Medicina Legal, a Jacinto, que trabajaba en la sección de Biología de la Escuela de Medicina Legal, y que había conocido el Instituto de Medicina Legal y, personalmente a Marañón. Durante los contactos que tuvimos con él en prácticas, nos transmitió alguna anécdota sobre la estancia de Marañón en la Escuela. Sobre ellas recuerdo la referencia a la energía con que ascendía al acudir por las tardes al laboratorio de Biología por aquellas incómodas escaleras de acceso, ágilmente, de dos en dos y de cuatro en cuatro, o la reprimenda que recibió en una ocasión del Prof. Maestre, en su despacho, porque había entrado silbando alegremente en el laboratorio, Jacinto recordaba que, desde aquel día, a Marañón no se le volvió a oír silbar. Es un buen reflejo del concepto que entonces se tenía del trabajo científico y la seriedad protocolaria con que se realizaba.

Se plantea entonces determinar la fecha en que Marañón estuvo al frente de la Sección de Biología. Se conoce perfectamente la secuencia de los directores de este laboratorio desde que el Instituto de Medicina Legal y Psiquiatría se transformó en Escuela de Medicina Legal. Cuando, en el año 29, se inaugura ésta, al frente de la misma estaba el Dr. Rafael Fraile, que luego sería director de la Escuela durante la Guerra, y González Bernal, su ayudante; terminada la Guerra, y con Fraile Ruiz de Quevedo depurado, González Bernal pasó a ser el jefe de la Sección y a éste le sucedió Moya Pueyo. En consecuencia, la fotografía de la que arranca este trabajo debe de corresponder a un período anterior. No puede ser la época de Marañón, recién graduado, trabajando con Hernando, ya que el Instituto se inaugura después, en 1914, Hernando gana la Cátedra de Terapéutica en 1912. Es llamativo que en esta fecha, Marañón realiza una publicación con Claudio Aznar, que demuestra que se encuentra vinculado a la institución. Claudio Aznar trabajó como profesor del Instituto de 1915 a 1927, el Instituto se transforma en Escuela en 1929, por lo tanto es en este período cuando Marañón debió hacerse cargo de la Sección de Biología.

No hemos podido encontrar documentación alguna de carácter oficial sobre el nombramiento de Marañón, pero dado el nivel científico que exigía Maestre para dirigir una Sección, parece más probable una fecha a partir del año 1922, en que Marañón ya es académico. Solamente un autor aporta una fecha y es Álvarez Sierra que, textualmente dice: «Marañón fue nombrado en 1912 profesor de la Escuela de Medicina Legal creada por el doctor don Tomás 
Maestre». Esta referencia resulta coherente ya que en 1909, Marañón se había formado con Ehrlich y era el primer médico español que había visto las historias clínicas de los sujetos tratados con neosalvarsan y el primero que trajo a España la reacción de Wasserman, por otro lado sorprende, dada su creatividad, que no hubiera publicado más en el campo medicolegal, encajando plenamente en su formación y vocación la Medicina Legal. Recordemos que Marañón se consideraba con una formación básicamente anatómica y fisiológica y con una tendencia y afición particular por la Psicología y la Psiquiatría.

Sirva como ejemplo el propio de Hernando. Tan pronto consiguió la cátedra, se presentó al Ministro de Instrucción Pública para solicitar que si se le dotaba de alguna mejora económica, más allá de las 5000 pesetas anuales de sus honorarios, se dedicaría exclusivamente a la docencia y la investigación. El ministro rechazó la propuesta, limitando con ello en gran medida las posibilidades de investigación de uno de los grandes y fecundos maestros de la Medicina Española. A lo largo de su vida mencionó varias veces esta historia amargamente, no por lo que le afectara personalmente sino por lo que de representativa tenía de la tónica general del país.

Otro testimonio, desgarrador, demostrativo, no solo de la situación del país, sino de la propia Medicina Legal, lo tenemos en palabras del propio Tomás Mestre, cuando, en 1927, despidió a Claudio Aznar y públicamente lamentó la marcha de aquel excelente investigador para ejercer en el medio rural, debido a que con lo que cicateramente le pagaba la Administración no podía nadie vivir, ni siquiera sobrevivir en Madrid, si no era a través de un pluriempleo agotador. Todavía el nombramiento del primero de los firmantes de este trabajo, como Jefe de la Sección de Investigación Criminal de la Escuela de Medicina Legal, en 1968, estaba dotado con veintidos mil pesetas al año (132 euros). Así Marañón, por aquel entonces tenía que alternar su trabajo en el Hospital Provincial de Madrid con la dirección del Hospital del Rey (1916-1924), con el trabajo en el Instituto de Medicina Legal por las tardes y con su consulta privada.

Esta situación le impresiona profundamente: y señala la ejemplaridad rigurosamente moral de la pobreza y el desprendimiento de Cajal, y en Claudio Bernard o en Pasteur que describe en su homenaje a Cajal pero que reitera en otras ocasiones. Ese planteamiento «no nos describe solo a Cajal, -escribe Pérez Gutiérrez-; nos describe también a Marañón... había unas condiciones perdurables a la que nuestro hombre se había vuelto muy sensible:...había que luchar encarnizadamente contra el medio, siempre comprometedor para la tarea creadora". El propio Marañón escribiría a propósito de la vida de Pasteur: "yo creo que el genio no se revela espontáneamente en la mayoría de los casos, sino después de una lucha encarnizada contra el medio, en la que sucumbe para la ciencia todo talento que no sea de orden excepcional... No seamos por lo tanto, ingratos con la pobreza y, sobre todo, no pongamos a la pobreza como pretexto para nuestra esterilidad. En los países sin ambiente científico como el nuestro, se padece de este achaque y se pide clamorosamente dinero... y está bien todo ello; pero sin pretender que de ahí arranque la ciencia verdadera» (III, 65). Escribió, en su discurso de ingreso de a Academia de Ciencias y en escritos sobre Cajal, este panorama desolador de la época que calificó de estéril: «En ese medio casi estéril la obra del maestro se alza, en verdad, no como una cima eminente de una cordillera, sino como solitaria montaña en la llanura».

La fecha de la fotografía, que motiva esta comunicación, surgió inopinadamente, al leer la sesión necrológica de Ara, personaje que esta presente en la fotografía. Ara estaba en la fotografía porque había puesto a punto su procedimiento de parafinización en la Escuela de Medicina Legal, y en la necrología que realizó Velázquez aparece una caricatura de este profesor en la que puede 
leerse: «Caricatura de D. Pedro Ara a tenor de la elegancia en el vestir y de su técnica de parafinización», realizada por el Dr. Emilio de la Peña, entonces estudiante de Medicina. Era una época en que D. Pedro Ara se había dejado una barbita en forma de «perilla». Está firmada E. Peña, fechada en 1925, «perilla» que precisamente luce en la fotografía del Instituto de Medicina Legal (Fotografia 3).

Según la costumbre del Instituto con los visitantes, se realizaba una fotografía oficial, que tomaba Blas Aznar, en la que se recogía la imagen de los profesores de la Escuela con quien había confraternizado, y esta foto da fe de la presencia de Marañón, de Ara, de Maestre e indirectamente de Blas Aznar, Jefes respectivamente de las Secciones de Biología, Tanatología, Psiquiatría e Instrumentación del Instituto de Medicina Legal, Toxicología y Psiquiatría como personajes altamente significativos de la investigación medicolegal del momento. Si es cierto el dato de Álvarez-Sierra 1912 a 1925 fue el periodo en que Marañón dedicó parte de su tiempo y esfuerzo, por las tardes, a la Medicina Legal. Un último dato parece confir-

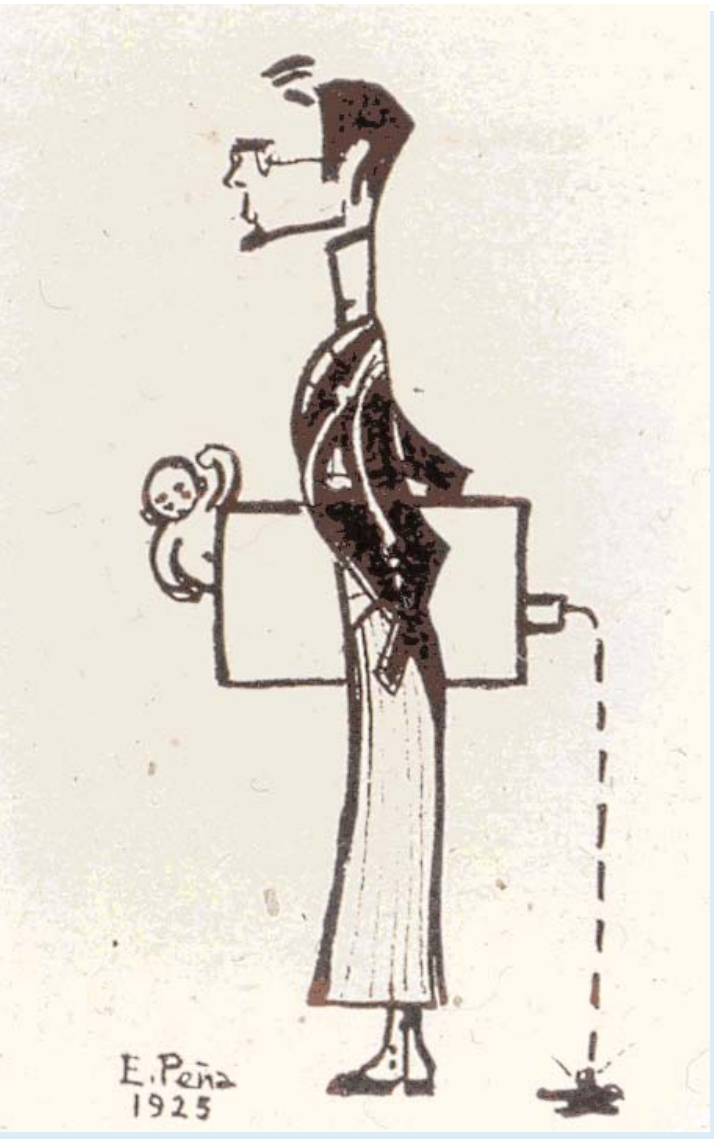

Fotografía 3 Caricatura de D. Pedro Ara a tenor de la elegancia en el vestir y de su técnica de parafinización", realizada por el Dr. Emilio de la Peña, entonces estudiante de Medicina (1925) mar que su estancia en el Instituto no fue mucho más allá; en 1924 renuncia a la dirección del Hospital del Rey y en 1925 funda el Instituto de Patología; es, por lo tanto, un momento trascendental de su vida en que Marañón se independiza, crea su escuela y rompe las ataduras administrativas con las instituciones de las que dependía anteriormente.

Por fin, gracias a la colaboración de Dña Ana Rocasolano pudimos encontrar documentación acreditativa no de su nombramiento pero si de su cese. En amable comunicación nos dijo:

En el Archivo General de la Universidad Complutense tenemos el expediente personal de Gregorio Marañón (AGUCM P-582, 7), en él aparece como único documento que le vincula al Instituto de Medicina Legal, Toxicología y Psiquiatría Central de España su cese como auxiliar del mismo, que le adjuntamos y que reproducimos fotográficamente (Fotografías 4 y 5).

Su cese voluntario, oficialmente se produjo en noviembre de 1925. 


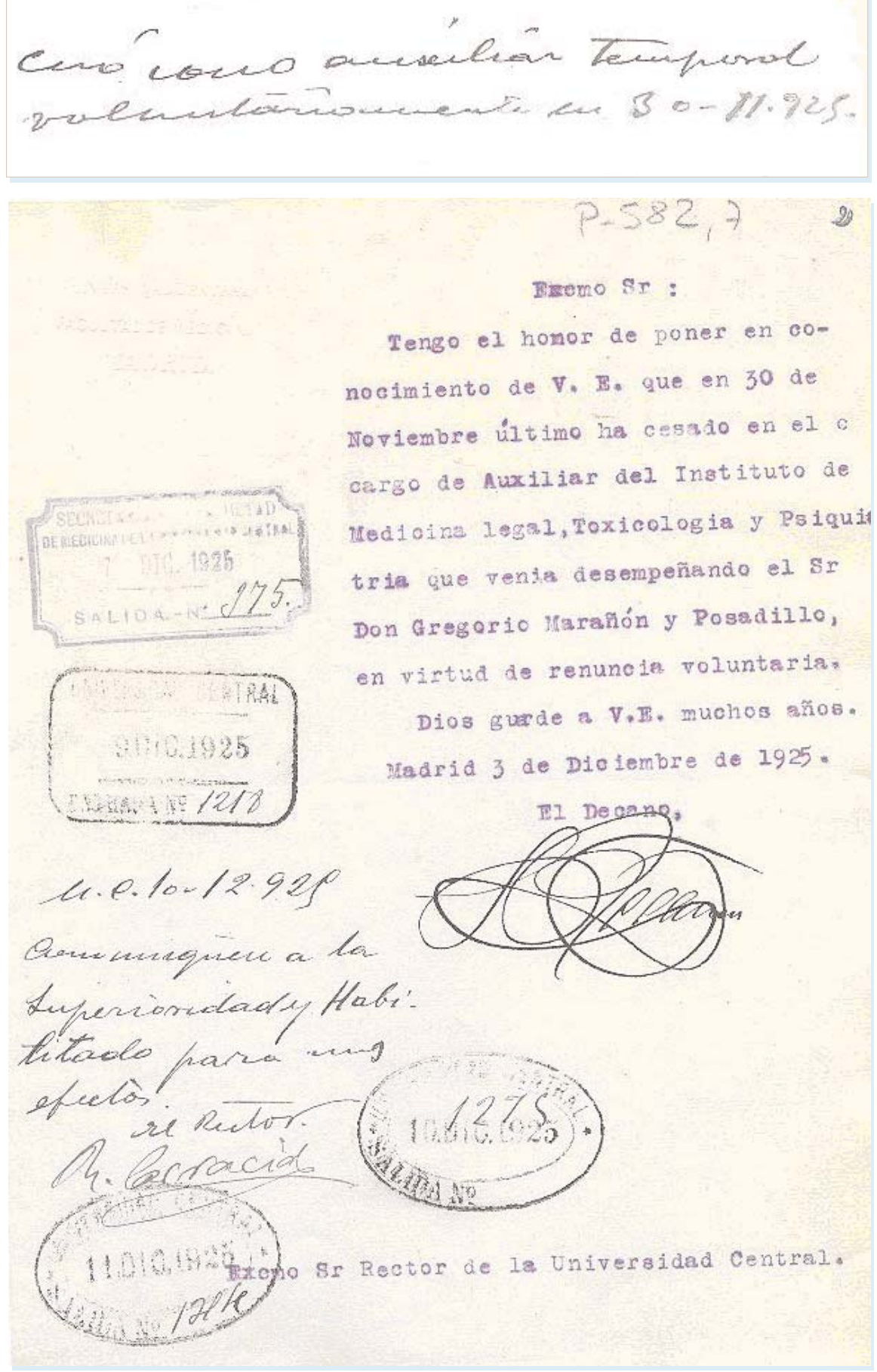

Fotografías 4 y 5 Expediente Gregorio Marañón en que se recoge su cese como auxiliar del Instituto de Medicina Legal, Toxicología y Psiquiatría Central de España

\section{Bibliografía}

1 ALVAREZ SIERRA, J. Diccionario de Autoridades Médicas. Ed. Nacional. Madrid, 1963. Pág.309.

2 AZNAR, C.; MARAÑÓN, G. La Acción Protectora de Diferentes Extractos de Órganos Contra la Estricnina. Bol. Soc. Esp. Biol., I, II; tomo I: 302 - 304, 1912.

3 Boletín de la Asociación Nacional de Médicos Forenses. 4 de agosto de 1969, Pág. 83.

4 CENTRO DE INVESTIGACIONES BIOLÓGICAS. Catálogo de Publicaciones del Prof. 
Gregorio Marañón Existentes en los Fondos de la Biblioteca del Centro de Investigaciones Biológicas. Comunidad de Madrid. Consejería de Salud. Consejo Superior de Investigaciones Científicas. Madrid, 1994.

5 GOMEZ - SANTOS, M. Vida de Gregorio Marañón. Plaza \& Janés. Barcelona, 1987.

6 GOMEZ - SANTOS, M. Gregorio Marañón Cuenta su Vida. Aguilar. Madrid, 1961.

7 GRANJEL, L.S. Gregorio Marañón, su Vida y su Obra. Guadarrama. Madrid, 1960.

8 JUDERÍAS, A. Obras Completas de Gregorio Marañón. Diez vol. Espasa Calpe. Madrid, 1966.

9 LAÍN, P.- Vida, Obra y Persona de Gregorio Marañón. En MARAÑóN, G.: Obras Completas. Tomo I. Espasa Calpe. Madrid, 1966.

10 MARAÑÓN, G. Prólogo. En PIGA, A.: Terapéutica de los Gaseados de Guerra. Rivadeneyra. Madrid, 1936.

11 MARAÑóN, G. La Medicina Legal y las Nuevas Orientaciones de la Ciencia. Justicia, I; 3: $5,1911$.

12 MARAÑón, G. Una Clasificación de los Homosexuales Desde el Punto de Vista de la Medicina Legal. En RIBEIRO, L.: Homosexualismo y Endocrinología. Prefacio. Arch. de Med. Leg. e Identificaçao. Río de Janeiro, 1936.

13 MARAÑON, G. Una generación y un hombre. Obras Completas. Tomo I. Prólogos, Espasa Calpe S. A. Madrid, 1966. Págs. 43-46.

14 PEREZ GutierREZ, F. La Juventud de Marañón. Trotta. Madrid, 1997.

15 SALINAS, M. Don Teófilo, cien años de Medicina Española. Biograma, I, 1: 6-30, 1973.

16 VELAZQUEZ, B. Sesión Necrológica en Homenaje al Prof. Ara (Pedro Ara Sarria) con motivo de su fallecimiento el 16 de septiembre de 1973. Arch. Fac. Med. Madrid, XXIV, 6: 315-330, 1973.

Correspondencia José Delfín Villalaín Blanco

San Blas $1,4^{\circ} \mathrm{B}$

28220. Majadahonda

Jose.d.villalain@uv.es 